\title{
Controlling ultracold Rydberg atoms in the quantum regime
}

\author{
Bernd Hezel, ${ }^{1, *}$ Igor Lesanovsky, ${ }^{2,+}$ and Peter Schmelcher ${ }^{1,3, t}$ \\ ${ }^{1}$ Physikalisches Institut, Universität Heidelberg, \\ Philosophenweg 12, 69120 Heidelberg, Germany \\ ${ }^{2}$ Institute of Electronic Structure and Laser, \\ Foundation for Research and Technology - Hellas, \\ P.O. Box 1527, GR-711 10 Heraklion, Greece \\ ${ }^{3}$ Theoretische Chemie, Institut für Physikalische Chemie, \\ Universität Heidelberg, INF 229, 69120 Heidelberg, Germany
}

(Dated: August 16, 2018)

\begin{abstract}
We discuss the properties of Rydberg atoms in a magnetic Ioffe-Pritchard trap being commonly used in ultracold atomic physics experiments. The Hamiltonian is derived and it is demonstrated how tight traps alter the coupling of the atom to the magnetic field. We solve the underlying Schrödinger equation of the system within a given $n$-manifold and show that for a sufficiently large Ioffe field strength the $2 n^{2}$-dimensional system of coupled Schrödinger equations decays into several decoupled multicomponent equations governing the center of mass motion. An analysis of the fully quantized center of mass and electronic states is undertaken. In particular, we discuss the situation of tight center of mass confinement outlining the procedure to generate a low-dimensional ultracold Rydberg gas.

PACS numbers: $32.60 .+\mathrm{i}, 33.55 . \mathrm{Be}, 32.10 . \mathrm{Dk}, 33.80 . \mathrm{Ps}$
\end{abstract}


Rydberg atoms possess remarkable properties. Although being electronically highly excited they can possess lifetimes of the order of milliseconds. Moreover, due to the large displacement of the valence electron and the atomic core they are highly susceptible to electric fields and therefore easily polarizable. The latter is impressively shown in ultracold Rydberg gases [1] where the mutual multipole interaction of two Rydberg atoms leads to a number of intriguing many body effects, such as resonant Förster transitions [2] and the dipole blockade mechanism [3, 4]. Their tunable electric dipole moment makes them also interesting candidates for the realization of a two-qubit quantum gate [5] which is a crucial ingredient for the realization of quantum information processing schemes. However, a prerequisite for realizing the latter in a coherent fashion is the availability of a well-controlled environment in which the Rydberg-Rydberg interaction takes place: Firstly one has to assure the Rydberg atoms to be individually addressable. Secondly, since the mutual interaction strength strongly depends on their relative displacement, a suitable way to spatially arrange the atoms has to be found.

There exist several proposals for building traps for Rydberg atoms suggesting the use of electric [6], optical [7] and magnetic fields [8]. The experimental feasibility of magnetic trapping has been shown recently by employing very strong magnetic fields [9]. Trapping in the quantum regime, which is indispensable to gain precise control over the atomic motion, however, could not yet be demonstrated. Properties of such quantized Rydberg states that emerge in a magnetic quadrupole trap have been theoretically discussed in Refs. [8]. The authors report on a strong confinement of the atomic center of mass (c.m.) with trapping frequencies of the order of $10 \mathrm{kHz}$. At the same time the electronic structure is, to a large extent, unchanged compared to the field-free case. Although the 3D magnetic quadrupole field is omnipresent in ultracold atom experiments it has one drawback. Due to the point of zero field strength at its center Majorana transitions are likely to happen thereby leading to loss from the trap. In Ref. [8] it was shown that by increasing the total angular momentum of the atom this problem can be overcome. However, for practical purposes it is desirable to have trapping at small or even zero center of mass angular momentum since this is the regime in which trapped ground state atoms are usually prepared.

In this letter we show that trapped and controllable Rydberg states can be achieved in a Ioffe-Pritchard (IP) trap. We discuss how the large size of the Rydberg atom modifies the coupling to the magnetic field in comparison to ground state atoms and demonstrate the 
feasibility of generating low dimensional Rydberg gases. Using the IP configuration is of particular relevance since it is usually employed for preparing ultracold atomic gases which can serve as the initial state for the production of trapped ultracold Rydberg atoms.

In the two-body picture the mutual interaction of the valence electron (particle 1) and the remaining core (particle 2) of the Rydberg atom can be modeled by an effective potential that exhibits short range properties describing the electron-core scattering and a long range Coulombic behaviour. States with large electronic angular momenta $l$, which represent the focus of the present investigation, probe almost exclusively the Coulombic tail of this potential. We do not account for relativistic effects such as spin-orbit coupling which for large $n, l$ are negligibly small compared to the energy shift due to the magnetic field of the IP trap [8, 10]. The interaction of the electronic and core charge with the magnetic field are taken into account via the minimal coupling. Following the above arguments and including the coupling of the nuclear and electronic magnetic moments to the external field our initial Hamiltonian reads

$$
\begin{aligned}
H_{\text {init }}= & \frac{1}{2 M_{1}}\left[\mathbf{p}_{1}-e \mathbf{A}\left(\mathbf{r}_{1}\right)\right]^{2}+\frac{1}{2 M_{2}}\left[\mathbf{p}_{2}+e \mathbf{A}\left(\mathbf{r}_{2}\right)\right]^{2} \\
& +V\left(\left|\mathbf{r}_{1}-\mathbf{r}_{2}\right|\right)-\boldsymbol{\mu}_{1} \cdot \mathbf{B}\left(\mathbf{r}_{1}\right)-\boldsymbol{\mu}_{2} \cdot \mathbf{B}\left(\mathbf{r}_{2}\right) .
\end{aligned}
$$

The IP field configuration is given by $\mathbf{B}(\mathbf{r})=B \mathbf{e}_{z}+\mathbf{B}_{\text {lin }}(\mathbf{r})+\mathbf{B}_{\text {quad }}(\mathbf{r})$ with the linear component $\mathbf{B}_{\text {lin }}(\mathbf{r})=G\left[x \mathbf{e}_{x}-y \mathbf{e}_{y}\right]$ and the quadratic component $\mathbf{B}_{\text {quad }}(\mathbf{r})=$ $\left.Q\left[2 z\left(x \mathbf{e}_{x}+y \mathbf{e}_{y}\right)+\left(x^{2}+y^{2}-2 z^{2}\right) \mathbf{e}_{z}\right)\right]$. In the following we consider the case of a dominant linear component and neglect $\mathbf{B}_{\text {quad }}(\mathbf{r})$. We therefore encounter a two-dimensional quadrupole field which is translationally invariant along the $z$-axis. Our corresponding vector potential reads $\mathbf{A}(\mathbf{r})=\mathbf{A}_{\mathrm{c}}(\mathbf{r})+\mathbf{A}_{\text {lin }}(\mathbf{r})$ with $\mathbf{A}_{\mathrm{c}}(\mathbf{r})=\frac{B}{2}\left[x \mathbf{e}_{y}-y \mathbf{e}_{x}\right]$ and $\mathbf{A}_{\text {lin }}(\mathbf{r})=G x y \mathbf{e}_{z}$. Next let us insert these expressions into the Hamiltonian (11) and introduce relative $\mathbf{r}$ and c.m. coordinates $\mathbf{R}$ with their respective momenta $\mathbf{p}$ and $\mathbf{P}$. In the absence of the external field one arrives at a decoupled c.m. and electronic motion. However, already in the presence of a homogeneous field this is not the case [11] and consequently terms coupling the c.m. and internal motion emerge. To simplify the latter in case of our inhomogeneous magnetic field configuration we apply the unitary transformation $U=\exp \left[i \frac{B}{2} \mathbf{e}_{z} \times \mathbf{r} \cdot \mathbf{R}\right]$ which eliminates couplings of the c.m. and the relative motion generated by the Ioffe field (atomic units are used throughout except when stated otherwise) [18]. Furthermore we neglect the diamagnetic terms, which is a very good approximation for laboratory field strengths and not too 
high principal quantum numbers $n$ (see ref. [8]), and keep only the leading order terms with respect to the inverse masses

$$
H_{\mathrm{IP}}=H_{A}+\frac{\mathbf{P}^{2}}{2 M_{2}}-\boldsymbol{\mu}_{2} \cdot \mathbf{B}(\mathbf{R})-\boldsymbol{\mu}_{1} \cdot \mathbf{B}(\mathbf{R}+\mathbf{r})+\mathbf{A}_{\operatorname{lin}}(\mathbf{R}+\mathbf{r}) \cdot \mathbf{p}
$$

Here $H_{A}=\frac{\mathbf{p}^{2}}{2}-\frac{1}{r}$ is the Hamiltonian of a hydrogen atom possessing the eigenfunctions $\left|n, l, m_{l}, m_{s}\right\rangle$ and energies $E_{n}=-\frac{1}{2} n^{-2}$. The following two terms of $H_{\mathrm{IP}}$ describe the c.m. motion of a point particle possessing the magnetic moment $\boldsymbol{\mu}_{2}$ in the presence of the field $\mathbf{B}$. This system has been thoroughly investigated in Refs. 12, 13]. Since the magnetic moments are connected to the corresponding spins $\mathbf{S}$ and $\boldsymbol{\Sigma}$ according to $\boldsymbol{\mu}_{1}=-\mathbf{S}$ and $\boldsymbol{\mu}_{2}=-\frac{g_{N}}{2 M_{2}} \boldsymbol{\Sigma}$, with $g_{N}$ being the nuclear $g$-factor, we neglect the term involving $\boldsymbol{\mu}_{2}$ in the following. The last two terms of $H_{\mathrm{IP}}$ couple the electronic and c.m. dynamics mediated by a spin-field and motionally induced coupling. We remark that the Hamiltonian (11) commutes with the $z$-component of the linear c.m. momentum $P_{z}$ being a direct consequence of the abovementioned translational invariance of the system along the $z$-axis. Hence the longitudinal motion can be integrated out employing plane waves $\left|K_{z}\right\rangle=\exp \left(-i K_{z} Z\right)$. In order to solve the eigenvalue problem of the resulting Hamiltonian, that depends on five spatial degrees of freedom, we assume the magnetic field not to couple adjacent $n$-manifolds. Estimating the energy level shift caused by the magnetic field according to $E_{\text {zee }} \approx B n$ this requirement is fulfilled if $\left|E_{n}-E_{n-1}\right| / E_{\text {zee }} \approx\left(B n^{4}\right)^{-1} \gg 1$. In this case we can consider each $n$-manifold separately and represent the Hamiltonian (2) in the space of the $2 n^{2}$ states spanning the given $n$-manifold. Neglecting the constant energy offset $E_{n}$ and introducing scaled c.m. coordinates $\left(\mathbf{R} \rightarrow \gamma^{-\frac{1}{3}} \mathbf{R}\right.$ with $\left.\gamma=G M_{2}\right)$ while scaling the energy unit via $\epsilon_{\text {scale }}=\gamma^{\frac{2}{3}} / M_{2}$ we eventually arrive at the working Hamiltonian

$$
\mathcal{H}=\frac{P_{x}^{2}+P_{y}^{2}}{2}+\boldsymbol{\mu} \cdot \mathbf{G}(X, Y)+\gamma^{-\frac{2}{3}} M_{2} \mathcal{H}_{r}
$$

with the effective magnetic field

$$
\mathbf{G}(X, Y)=X \mathbf{e}_{x}-Y \mathbf{e}_{y}+\gamma^{-\frac{2}{3}} M_{2} B \mathbf{e}_{z}
$$

where $\boldsymbol{\mu}$ and $\mathcal{H}_{r}$ are the $2 n^{2}$-dimensional matrix representations of the operators $1 / 2[\mathbf{L}+2 \mathbf{S}]$ and $H_{r}=\mathbf{A}_{\text {lin }}(\mathbf{r}) \cdot \mathbf{p}+\mathbf{B}_{\text {lin }}(\mathbf{r}) \cdot \mathbf{S}$, respectively. Here we have introduced the orbital angular momentum operator $\mathbf{L}=\mathbf{r} \times \mathbf{p}$.

The Hamiltonian (3) can be interpreted as follows: The first two terms describe the dynamics of a neutral atom in a IP trap under the assumption that the coupling of the atom 
to the field is given by the Zeeman energy $E_{z}=1 / 2[\mathbf{L}+2 \mathbf{S}] \cdot \mathbf{G}(X, Y)$. One encounters a similar coupling term also for 'point-like' ground state atoms [13] where, however, the generically strong hyperfine coupling leads to the fact that $\boldsymbol{\mu}$ is proportional to the total spin F. The distinct feature of the Hamiltonian (3) is the appearance of the last term which accounts for the finite size of the Rydberg atom. This term scales according to $\gamma^{-\frac{2}{3}} G M_{2} n^{2}=\gamma^{\frac{1}{3}} n^{2} \approx \gamma^{\frac{1}{3}}\langle r\rangle$. Since $\gamma^{-\frac{1}{3}}$ can be regarded as a length unit for the c.m. wave function we find this term to be particularly important if $\langle r\rangle \approx\langle R\rangle$, i.e. if the size of the atom and the size of the c.m. state become comparable. In a typical macroscopic IP trap [9] the c.m. wave functions are very extended and the energy spacing between the c.m. states is small. Hence, in this 'classical' regime $\mathcal{H}_{r}$ has little effect and can be neglected. The situation changes for tighter IP traps which can, for example, be realized by so-called atom chips 15$]$. Here the micro structured wires allow for the generation of traps for which the size of the c.m. ground states are of the order of several $100 \mathrm{~nm}$ and become therefore comparable to the typical size of Rydberg atoms.

In order to solve the Schrödinger equation belonging to the Hamiltonian (3) we employ an adiabatic separation of the electronic and the c.m. motion. To this end a unitary transformation $U(X, Y)$ which diagonalizes the last two (matrix) terms of the Hamiltonian, i.e. $U^{\dagger}(X, Y)\left(\boldsymbol{\mu} \cdot \mathbf{G}(X, Y)+\gamma^{-\frac{2}{3}} M_{2} \mathcal{H}_{r}\right) U(X, Y)=E_{\alpha}(X, Y)$, is applied. Since $U(X, Y)$ depends on the c.m. coordinates the transformed kinetic term involves non-adiabatic (off-diagonal) coupling terms which we will neglect in the following. We are thereby lead to a set of $2 n^{2}$ decoupled differential equations governing the adiabatic c.m. motion within the individual two-dimensional energy surfaces $E_{\alpha}$, i.e. the surfaces $E_{\alpha}$ serve as potentials for the c.m. of the atom. Figure 1 shows an intersection through a subset of these surfaces for the case of ${ }^{87} \mathrm{Rb}$ in a IP trap with a gradient $G=20 \mathrm{~T} / \mathrm{m}$ and a Ioffe field strength $B=0.01 \mathrm{G}$. One immediately notices the large number of avoided crossings between the adiabatic potential surfaces. Here non-adiabatic transitions mediated by the (neglected) off-diagonal coupling terms of the kinetic energy are likely to occur. The uppermost surface, however, does not exhibit such avoided crossings and is a possible candidate in order to achieve stable trapping. According to our findings this surface is predominantly formed by the electronic state possessing the largest possible orbital angular momentum in the $n$-manifold under consideration. The corresponding quantum defects and relativistic corrections are therefore tiny which a posteriori justifies their neglect. 


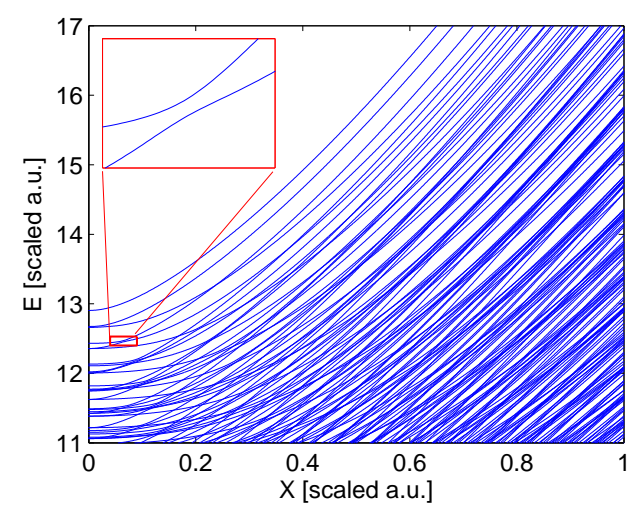

FIG. 1: Cut through the adiabatic potential surfaces of the $n=30$-manifold $\left({ }^{87} \mathrm{Rb}, G=20 \mathrm{~T} / \mathrm{m}\right.$, $B=0.01 \mathrm{G})$. The high density of states is clearly visible. The uppermost potential surface is predominantly formed by the atomic state with largest angular momentum. This particular surface is clearly separated from the next lower ones whereas all other surfaces exhibit a number of avoided crossings (see the magnified view in the inset) at which non-adiabatic inter-surface transitions are likely to occur.

The actual appearance of the potential surfaces depends on both, the gradient and the strength of the homogeneous Ioffe field. In figure 1 the latter is comparatively small such that for small displacements from the coordinate center its field strength is easily surpassed by that of the gradient field. The different symmetry properties of the both fields lead to a rearrangement of the energy surfaces finding its expression in the large number of avoided crossings. We now turn to the case where the Ioffe field is large compared to that of the gradient field, i.e. $\gamma^{-\frac{2}{3}} M_{2} B \gg 1$. This however can hold true only in the vicinity of the trap center. We assume this region to be sufficiently large such that at least a few low-lying c.m. states are localized here. In this case the term $\boldsymbol{\mu} \cdot \mathbf{G}(X, Y)$ will dominate the Hamiltonian (3). We now diagonalize this term by employing the transformation $U_{D}(X, Y)=\exp \left[i\left(L_{z}+S_{z}\right) \phi\right] \exp \left[i\left(L_{y}+S_{y}\right) \beta\right]$ with $\phi=\arctan [Y / X]$, $\cos \beta=\gamma^{-\frac{2}{3}} M_{2} B|\mathbf{G}(X, Y)|^{-1}$ and $\sin \beta=-\left(X^{2}+Y^{2}\right)|\mathbf{G}(X, Y)|^{-1}$. This yields the adiabatic energy surfaces

$$
E_{\alpha}=U_{D}^{\dagger}(X, Y) \boldsymbol{\mu} \cdot \mathbf{G}(X, Y) U_{D}(X, Y)=\frac{1}{2}\left(L_{z}+2 S_{z}\right)|\mathbf{G}(X, Y)|
$$

being characterized by the quantum numbers of $L_{z}$ and $S_{z}$ which are $m_{l}$ and $m_{s}$, respectively. The energetically highest surface is assumed for $m_{l}=n-1$ and $m_{s}=1 / 2$. The next lower 


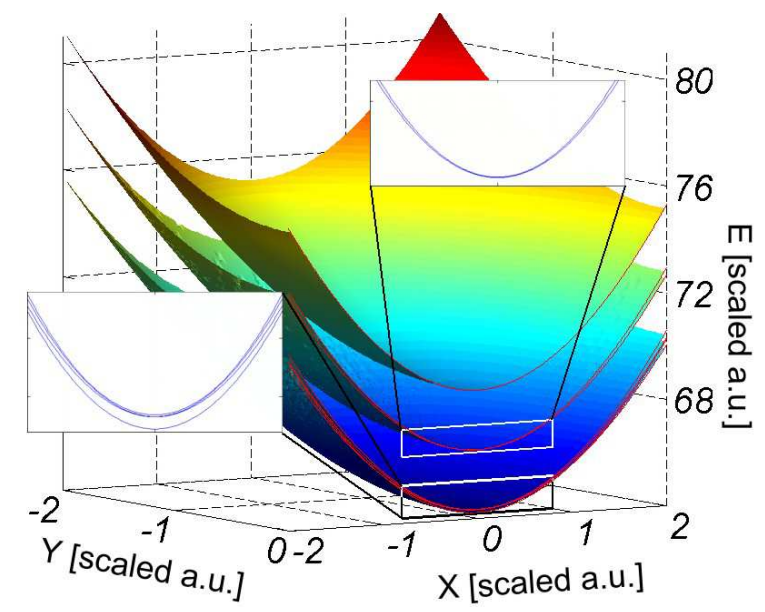

FIG. 2: Surface plot of the the seven uppermost energy surfaces of the $n=30$-manifold $\left({ }^{87} \mathrm{Rb}\right.$, $G=20 T / m B=0.1 G)$. Clearly the grouping into three multiplets whose mutual distance is given by $\gamma^{-\frac{2}{3}} M_{2} B$ is visible. While the uppermost manifold consists only of a single surface the next lower ones show an approximate twofold and fourfold degeneracy. A magnified view of them is provided in the insets.

one is twofold degenerate and the following one shows a fourfold degeneracy (see figure 21). The energy gap between these degenerate multiplets is given by $\triangle E \approx \gamma^{-\frac{2}{3}} M_{2} B$ and can hence be continuously varied by tuning the Ioffe field strength. In the present regime the term $\gamma^{-\frac{2}{3}} M_{2} \mathcal{H}_{r}$ can be considered as a perturbation since its energetic contribution is much smaller than $\triangle E$. The correction to the uppermost surface is zero whereas $\mathcal{H}_{r}$ couples the surfaces of the energetically lower lying degenerate multiplets. To study the dynamics of the multi-component c.m. wave function within these coupled potential surfaces constitutes a very interesting problem. In the present investigation, however, we will focus exclusively on the uppermost non-degenerate surface.

The explicit knowledge of $U_{D}(X, Y)$ allows for an analytical calculation of the nonadiabatic couplings between any of the potential surfaces arising from the kinetic energy term. Our findings show them to be proportional to $\gamma^{\frac{2}{3}}\left(M_{2} B\right)^{-1}=(\triangle E)^{-1}$. For a sufficiently large Ioffe field strength we can thus safely employ the adiabatic approximation, i.e. neglect the non-adiabatic coupling between the uppermost and the next lower surface. In order to obtain the quantized c.m. states we solve the scalar Schrödinger equation $\left[1 / 2\left(P_{x}^{2}+P_{y}^{2}\right)+E_{\alpha}(X, Y)-\epsilon_{\nu}\right] \Psi_{\nu}(X, Y)=0$ in the uppermost potential surface which we denote by $\alpha=2 n^{2}$. For low c.m. excitations the potential surfaces are approximately har- 


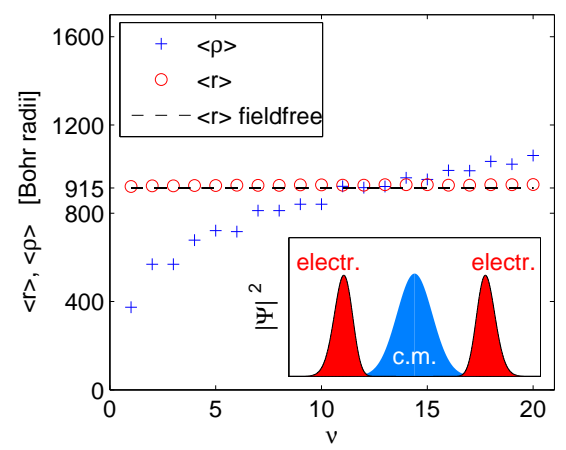

FIG. 3: Expectation value of the radii $\rho=\sqrt{X^{2}+Y^{2}}$ and $r$ of the c.m. and electronic wave function in the IP trap $\left({ }^{87} \mathrm{Rb}, G=100 \mathrm{~T} / \mathrm{m}, B=0.1 G\right) . \nu$ labels the c.m. quantum states within the uppermost adiabatic potential surface. While $\langle\rho\rangle$ is increasing $\langle r\rangle$ remains approximately constant at its field free value. For small degrees of c.m. excitation the c.m. state is even stronger localized than the valence electron $(\langle\rho\rangle\langle\langle r\rangle)$. Moreover, since the electron is found in a high angular momentum state its radial uncertainty $\Delta r$ is small. Thus a scenario where the c.m. and the electronic wave function do not overlap is possible as sketched in the inset.

monic and thus the energies are in reasonably good agreement to those of a two-dimensional isotropic harmonic oscillator with a $n$-dependent frequency $\omega=G \sqrt{\frac{n}{2 B M_{2}}}$ (in atomic units). Hence, by choosing high gradients and an appropriate tuning of the Ioffe field strength $B$ very tightly confining traps for highly excited atoms can be obtained. Such a situation is depicted in figure 3 where we show the expectation values of the radii $\rho=\sqrt{X^{2}+Y^{2}}$ and $r$ for the c.m. and electronic wave function versus the degree of excitation of the c.m. motion $\nu$. In the presented case the confinement gives rise to a trap frequency of approximately 1.4 MHz. In this regime the size of the c.m. state characterized by $\langle\rho\rangle$ is even smaller than the electronic cloud, i.e. the c.m. wave function is stronger localized than the valence electron. On the other hand the expectation value $\langle r\rangle$ for the electron remains nearly constant possessing the corresponding field free value as the degree of excitation of the c.m. increases. This indicates that in spite of the strong localization of the c.m. the electronic structure of the atom is barely changed compared to the field free case. This observation has been backed up by calculating further electronic properties, such as the expectation values of $\mathbf{L}^{2}$ which also barely differ from their corresponding field free values. As previously indicated we find the electron in the highest angular momentum state $(l=n-1)$ which possesses the smallest radial uncertainty $\Delta r$ for given $n$. Due to this fact it is possible that the c.m. and 
the electronic wave function may not even overlap (see inset of figure 3). This novel regime opens up the possibility to control Rydberg atoms in the quantum regime and might pave the way to study many-body effects in low-dimensional ultracold Rydberg gases [14].

In order to study the latter, ultracold atoms confined in a tight atom chip trap 15] can be transferred into high- $l$ Rydberg states by imposing suitable optical and radio frequency fields (see refs. 16, 17] and refs. therein). Since the electronic structure is barely affected even for tight c.m. confinement the Rydberg atoms keep their well-known properties such as long radiative lifetimes and electric dipole moments.

I.L. acknowledges support by the European Community and its 6th Community Frame under the program of scholarships 'Marie Curie'. P.S. acknowledges financial support by the Deutsche Forschungsgemeinschaft.

* hezel@physi.uni-heidelberg.de

$\dagger$ igor@iesl.forth.gr

$\ddagger$ Peter.Schmelcher@pci.uni-heidelberg.de

[1] I. Mourachko et al, Phys. Rev. Lett. 80, 253 (1998)

[2] T. H. Gallagher et al, Phys. Rev. A 25, 1905 (1982)

[3] M. D. Lukin et al , Phys. Rev. Lett. 87, 037901 (2001)

[4] D. Tong et al, Phys. Rev. Lett. 93, 063001 (2004)

[5] D. Jaksch et al, Phys. Rev. Lett. 85, 2208 (2000)

[6] P. Hyafil et al, Phys. Rev. Lett. 93, 103001 (2004)

[7] S.K. Dutta et al, Phys. Rev. Lett. 85, 5551 (2000)

[8] I. Lesanovsky and P. Schmelcher, Phys. Rev. Lett. 95, 053001 (2005); Phys. Rev. A 72, 053410 (2005)

[9] J.-H. Choi et al, Phys. Rev. Lett. 95, 243001 (2005)

[10] H. A. Bethe and E. Salpeter Quantum Mechanics of One- and Two-Electron Atoms, New York Plenum (1977)

[11] P. Schmelcher and L.S. Cederbaum, Phys. Lett. A 164, 305 (1992); O. Dippel, P. Schmelcher and L.S. Cederbaum, Phys. Rev. A 49, 4415 (1994)

[12] I. Lesanovsky and P. Schmelcher, Phys. Rev. A 70, 063604 (2004); Phys. Rev. A 71, 032510 
(2005)

[13] J. Bill, M. Trappe, I. Lesanovsky and P. Schmelcher, Phys. Rev. A 73, 053609 (2006)

[14] T. J. Carroll, K. Claringbould, A. Goodsell, M. J. Lim, and M. W. Noel, Phys. Rev. Lett. 93, $153001(2004)$

[15] R. Folman et al, Adv. At. Mol. Opt. Phys. 48, 263 (2002)

[16] L. Chen et al, J. Phys. B 26, L437 (1993)

[17] R. Lutwack et al, Phys. Rev. A 56, 1443 (1997)

[18] $\hbar=1, M_{1}=m_{e}=1, a_{0}=1$ : The magnetic gradient unit then becomes $b=1 a . u$. $=$ $4.44181 \cdot 10^{15} \frac{\mathrm{T}}{\mathrm{m}}$. The magnetic field strength unit is $B=1 a . u .=2.35051 \cdot 10^{5} \mathrm{~T}$ 